\title{
Pitfalls of Atrial Advancement Using a Ventricular Extra-stimulus During Supraventricular Tachycardia
}

Jeong-Wook Park, CEPS ${ }^{1}$; Sung-Hwan Kim, $\mathrm{MD}^{1,2}$; Yong-Seog Oh, MD, $\mathrm{PhD}^{1,2}$; Chun Hwang, MD, FHRS ${ }^{3}$

'Cardiocerebrovascular Center, Seoul St. Mary’s Hospital, Seoul, Korea; ${ }^{2}$ Division of Cardiology, Department of Internal Medicine, College of Medicine, The Catholic University of Korea, Seoul, Korea; ${ }^{3}$ Utah Valley Regional Medical Center, Provo, Utah, USA

Received: February 3, 2015

Accepted: March 28, 2016

Correspondence: Yong-Seog Oh, MD, PhD

Division of Cardiology, Department of Internal Medicine, College of Medicine, Seoul St. Mary's

Hospital, The Catholic University of Korea, 222 Banpo-daero, Seocho-gu, Seoul, Republic of Korea, 137-701

Tel: +82-2-3779-1325 Fax: +82-2-3779-1374

E-mail: oys@catholic.ac.kr

Copyright (C) 2016 The Official Journal of Korean Heart Rhythm Society Editorial Board \& MMK Co., Ltd.

\begin{abstract}
The delivery of single His-refractory ventricular extra-stimulus during supraventricular tachycardia is useful to identify the mechanism of the tachycardia. We present the different responses based on the ventricular extra-stimulus site. Our findings demonstrate that the atrial activation via an accessory pathway was not advanced based on the ventricular pacing site. Therefore, atrioventricular tachycardia could masquerade as atrioventricular nodal reentrant tachycardia.
\end{abstract}

Key Words: - Cardiac Mapping - Tachycardia, Supraventricular - Tachycardia, Atrioventricular Nodal Reentry

\section{Introduction}

The differentiation of the retrograde conduction pathway is essential for the diagnosis of paroxysmal supra ventricular tachycardia (PSVT). An eccentric retrograde atrial activation sequence during the tachycardia could give rise to a diagnosis of accessory pathway (AP) conduction. However, in the case of PSVT, which exhibits a concentric retrograde atrial activation sequence, it is confusing to identify whether the retrograde conduction pathway is through the atrioventricular $(\mathrm{AV})$ node or via an AP. Pacing maneuvers are needed to differentiate the retrograde conduction pathway during the tachycardia. The most common pacing maneuver used to identify AP conduction is the delivery of a single His-refractory ventricular extra-stimulus (VES) during the tachycardia. The main pitfall of this maneuver is the distance from the remote pacing site to the AP, such as a VES from the right ventricular (RV) apex to the left lateral AP. A remote pacing site will not be able to pre-excite the ventricular myocardium near the AP, and as a result, the AP conduction will not be manifested. However, despite the relatively short distance, this pitfall arose in our case and involved a VES from the RV apex to the right antero-septal AP. With this case report, we would like to propose a hypothetical mechanism to explain this finding.

\section{Case}

A 19-year-old man underwent an electrophysiological study due to palpitations and chest discomfort. His resting 12-lead electrocardiogram was normal without any pre-excitation and the baseline atrio-His and His-ventricular intervals were 110 and 54 

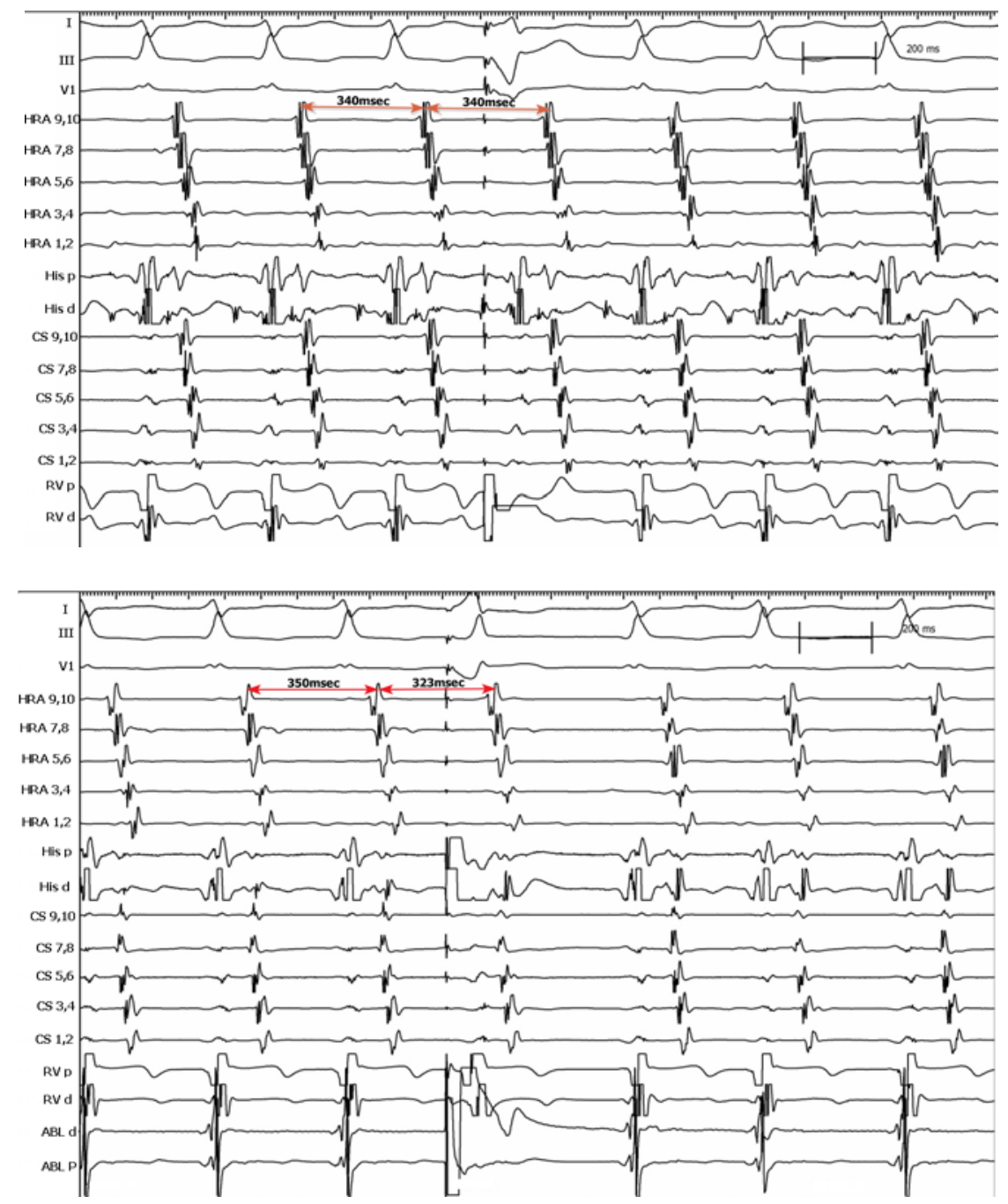

Figure 1. The high right atrial $(H R A)$ catheter was located from a high $(9,10)$ to low $(1,2)$ lateral area of the right atrium. The His catheter was located in the His region. The right ventricular (RV) catheter was located in the RV apex. (A) A ventricular extra-stimulus (VES) was introduced via the distal RV electrode when the bundle of His was refractory, which did not reset the supraventricular tachycardia. (B) The ablation catheter was located in the right ventricular para-Hisian region. A VES was introduced from the distal electrode of the mapping catheter when the bundle of His was refractory, which altered the subsequent atrial timing.

ABL, ablation; CS, coronary sinus; d, distal; His, His bundle; HRA, high right atrium; p, proximal; RV, right ventricle.

ms, respectively. The earliest retrograde atrial activation during RV pacing occurred in the midline without any decremental property. Dual atrioventricular nodal physiology was absent. A short RP interval tachycardia was induced by an atrial extra-stimulus after isoproterenol ( $2 \mu \mathrm{g} / \mathrm{min})$ administration. The retrograde atrial activation sequence during the tachycardia was the same as that during RV pacing. A single VES was delivered to the RV apex
(Figure 1A), and the RV antero-septum (Figure 1B), respectively, during the tachycardia when the His bundle was refractory. As a result, only the RV antero-septal VES altered the subsequent atrial timing. The tachycardia was terminated during every attempt at entrainment from the RV. Based on these maneuvers, what was the most likely diagnosis? 


\section{Discussion}

The differential diagnosis of a short RP interval narrow complex tachycardia with the earliest atrial activation in the midline includes junctional ectopic tachycardia (JET), atrioventricular nodal re-entrant tachycardia (AVNRT), and atrioventricular re-entrant tachycardia (AVRT) using a septal AP. In the present case, a His-refractory VES, over a wide range of the coupling interval, delivered to the RV apex during the tachycardia reproducibly failed to alter the subsequent atrial timing (Figure 1A). Such an observation is often thought to exclude the participation of an AP, and narrows the diagnosis to AVNRT or JET. Delivering a single His-refractory VES during the tachy cardia is the most common pacing maneuver used to identify extra-nodal conduction. A limitation of this approach is that several factors can contribute to the manifestation of the AP conduction, including a pacing site remote from the AP, the local ventricular refractory period, and the tachycardia cycle length. ${ }^{1}$ If the pacing site is remote from the circuit, such as in the contralateral chamber from the AP, the paced VES may not have enough time to reach the AP and affect the AVRT circuit when delivered late enough to be His-refractory. For example, failing to demonstrate a left lateral AP by a VES from the RV apex frequently occurs in clinical electrophysiological studies. On the contrary, the development of bundle branch block (BBB) on the same side as the $\mathrm{AP}$ would facilitate validating the $\mathrm{AP}$ conduction. BBB led to the conduction being delayed in the bundle branch and subsequently, in the ventricular myocardium on the same side as the AP, which lengthened the size of the reentrant circuit. As a result, a His-refractory VES could easily reach the AP before the arrival of the ventricular activation over the normal conduction pathway. In Figure 2, the QRS morphology during sinus rhythm and the tachycardia slightly differed, and was pronounced in V1 and resembled an incomplete right bundle branch block (RBBB) pattern. The tachycardia appeared to prolong the relative refractory period (RRP) of the right bundle branch ( $R B B$ ) compared to the left bundle branch (LBB), which led to conduction slowing in the RV and manifested as an incomplete RBBB pattern on the ECG. Thus, the development of an incomplete $\mathrm{RBBB}$ during the tachycardia in our case would have clearly demonstrated a right-sided AP conduction by a VES from the RV.
In this case, however, despite the relatively short distance to the AP, accompanied by the development of an ipsilateral BBB, a VES from the RV apex was unable to discriminate the AP conduction, which was through a right anteroseptal AP. What mechanism would lead to such a situation? The hypothetical plausible mechanism would be the presence of a mid-ventricular exit of the $\mathrm{RBB}$, or the presence of a left septal fascicle branching from the LBB. Depending on the ventricular activation near the septal AP via the $\mathrm{RBB}$ or the left septal fascicle, we hypothesized two case scenarios.

During a normal QRS, the earliest ventricular activation occurs in the ventricular septum, and if a septal fascicle is present, the activation may occur via the left septal fascicle. ${ }^{2}$ Thus, if the ventricular myocardium near the septal AP is activated via the left septal fascicle, and if they are closely located, it would appear to act as a relatively self-contained circuit. Consequently, the retrograde limb of the reentrant circuit of the AVRT might be decreased. In contrast, if the ventricular myocardium near the AP is activated via the $\mathrm{RBB}$, such as with a relatively lateral $\mathrm{AP}$, the orthodromic wavefront should pass the terminalis of the RBB. As a result, the

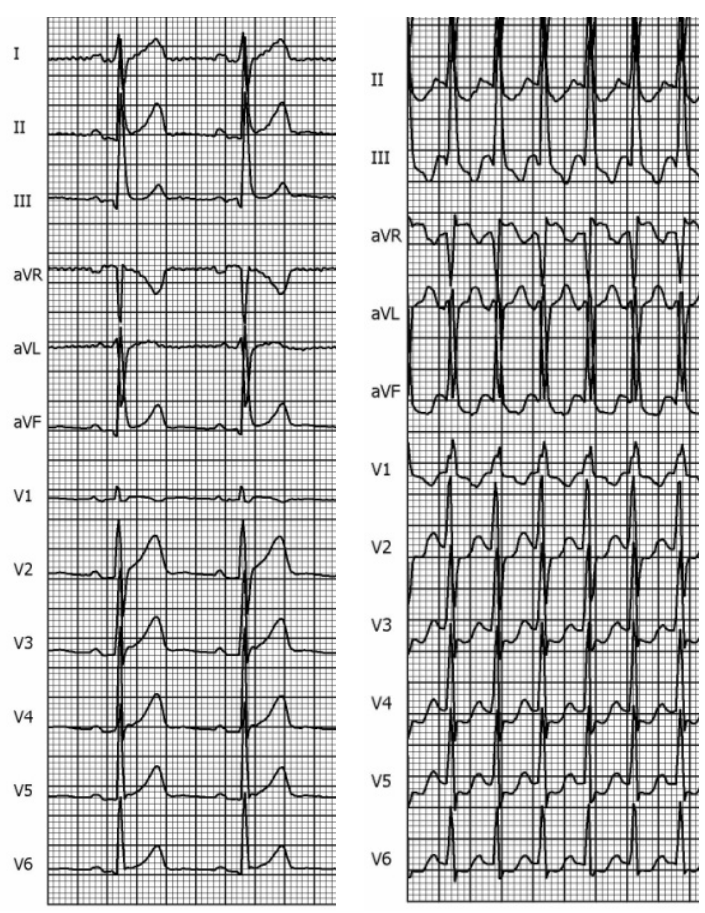

Figure 2. The QRS morphology during sinus rhythm and the tachycardia differed slightly, which is evident in V1 and resembles an incomplete right bundle branch block (RBBB) pattern. 

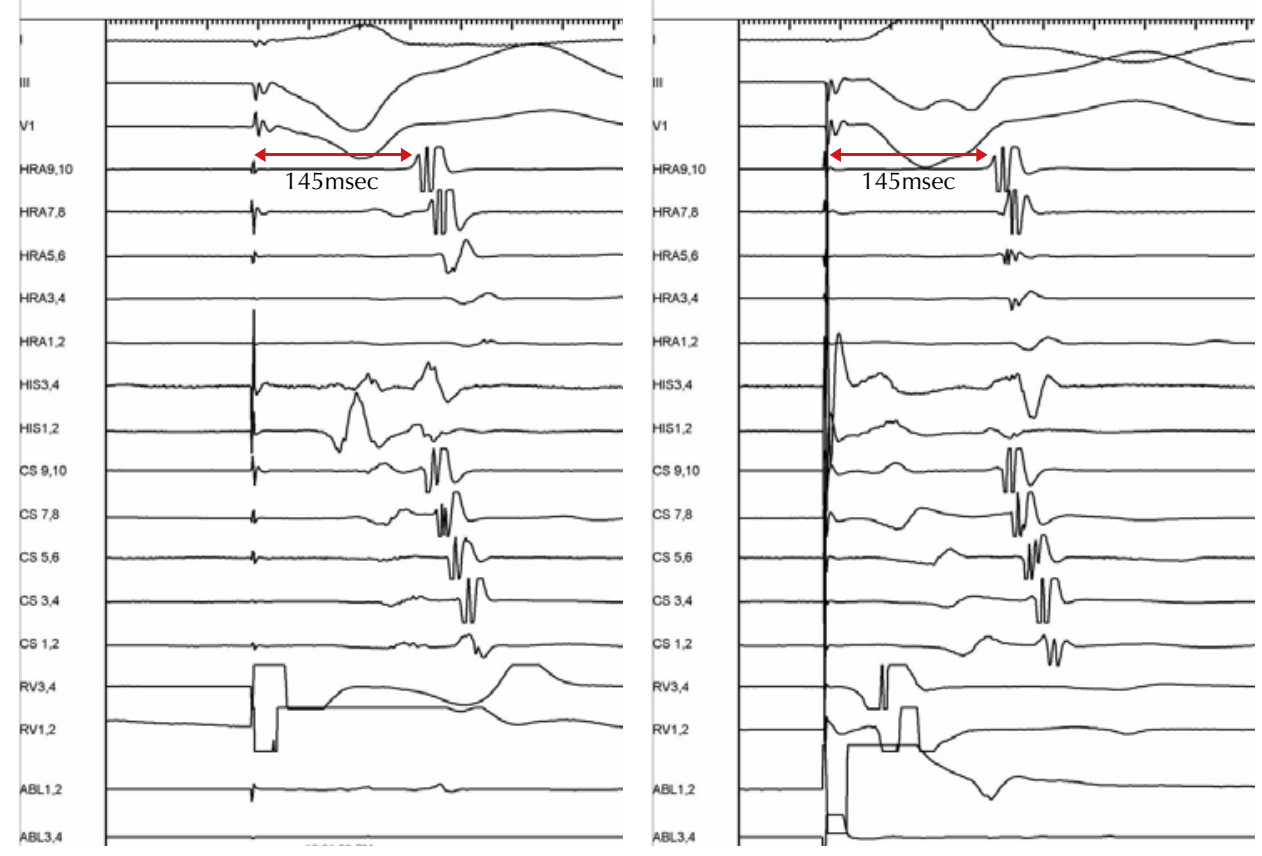

Figure 3. The diagnostic catheter is located at the same site as Figure 1B. In the left panel, ventricular pacing was performed from the RV apex. In the right panel, ventricular pacing was performed from the RV base. After elimination of the AP conduction, the paced VA interval from the RV apex and the RV base was the same. $A B L$, ablation; CS, coronary sinus; His, His bundle; HRA, high right atrium; RV, right ventricle.

retrograde limb of the reentrant circuit of the AVRT might be increased, rather than the AVRT using a septal AP. Because the $\mathrm{RBB}$ terminalis is located near the RV apex, the orthodromic wavefront using the RBB as the antegrade conduction pathway should go down to the RV apex. However, the orthodromic wavefront of the septal AP, which is activated from the left septal fascicle, does not need to go down to the RV apex to sustain the reentry, because the orthodromic wavefront would turn around at the ventricular basal septum where the exit of the left septal fascicle is located. Thus, in the case of the AVRT using a septal AP, which is activated via the septal fascicle, the RV apex can be excluded from the critical re-entrant circuit. In turn, a VES from the RV apex may not reach to the reentrant circuit during the Hisrefractory timing. In addition, if the myocardium near the AP were to be activated by the fascicle branching from the LBB, the $\mathrm{RBBB}$ would not improve the diagnostic utility of a Hisrefractory VES from the RV.

If the myocardium near the AP were to be activated by the $\mathrm{RBB}$, in our case, this would be explained by the presence of a basal exit of the RBB. The RBB is a limb of the reentrant circuit in cases of AVRT using a right-sided AP. The conventional anatomical location of the RBB determines the low turn around point of the reentry circuit, and as the electrical impulse passes from the RBB terminalis, it goes up to the RV base where the AP is located. The RBB terminalis is usually located near the RV apex, though the exact location is unknown and is subject to anatomical variation. ${ }^{3}$ The low turn around point of the reentry circuit can vary depending on the anatomical location of the $\mathrm{RBB}$ terminalis. Thus, if a patient has a mid-ventricular exit of the RBB terminalis, the low turnaround point will be shifted to the mid-ventricle and the RV apex can be excluded from the critical reentrant circuit. This hypothesis would be supported by the fact that the paced VA interval from the RV base was the same as the RV apex after elimination of the AP conduction in this case (Figure 3). The earlier engagement of the HPS with the RV mid-ventricle, rather than the RV apex, may explain such a result. ${ }^{4}$ In this circumstance, it would be difficult to invade the reentrant circuit from the RV apex during the His-refractory timing. A VES above the lower turnaround point, such as the RV summit, would have a greater chance to enter the reentrant circuit. ${ }^{5}$ 
For the reason mentioned above, we delivered a His-refractory VES to the RV anteroseptal region, which propagated to the ventricular myocardium near the $\mathrm{AP}$ (Figure $1 \mathrm{~B}$, the timing of the local myocardium near the His bundle was advanced), and then, the paced impulse advanced to the next atrial activation with a fused QRS morphology (Figure 1B). The alteration of the subsequent atrial timing by the VES with a fused QRS morphology suggested that the collision between the stimulated antidromic wavefront and orthodromic wavefront from the previous beat occurred in the ventricular myocardium. This finding never occurs in AVNRT and suggests a diagnosis of an AVRT. Ventricular overdrive pacing (VOP) was also performed at the RV apex and septum. However, the tachycardia terminated with every attempt to entrain it. Thus, we could not measure the post-pacing interval from the RV apex or septum. However, at the beginning of the VOP from the RV, the tachycardia resetting occurred before a stable paced QRS morphology, which is also compatible with the presence of AP conduction. ${ }^{6}$ A merged VA potential was observed at the right anteroseptum during $\mathrm{RV}$ pacing and the retrograde VA conduction disappeared during the application of radio-frequency energy to the region within 4 seconds. Of course, as described above, this did not rule out an AVNRT with a bystander AP. However, the observation that no further SVT could be induced by programmed stimulation with no echo beats after eliminating the AP conduction, and the absence of a dual atrioventricular nodal physiology supported participation of an AP in the clinical tachycardia.

In summary, the diagnosis was an orthodromic AVRT using a right anteroseptal AP. Although the RV apex was located ipsilateral to the reentrant circuit, a His-refractory VES from the $\mathrm{RV}$ apex did not discriminate the AP conduction. The most plausible explanation for this finding is the presence of a left septal fascicle and the presence of a mid-ventricular exit of the RBB terminalis. This case demonstrated the importance of the ventricular pacing site corresponding to the AP location in order to evaluate the operative AP conduction. To improve the diagnostic utility of a single His-refractory VES during the tachycardia, the VES should be delivered to the ventricular myocardium in the vicinity of the earliest atrial activation site.

\section{References}

1) Josephson ME. Clinical Cardiac Electrophysiology. 4th ed. Philadelphia: Lippincott Williams \& Wilkins; 2008. p.175-284.

2) Demoulin JC, Kullbertus HE. Histological examination of concept of left hemiblock. Br Heart J. 1972;34:807-814.

3) Massing GK, James TN. Anatomical configuration of the His bundle and bundle branches in the human heart. Circulation 1976;53:609-621.

4) Derval N, Skanes AC, Gula LJ, Gray C, Denis A, Lim HS, Krahn AD, Yee R, Sacher F, Haïssaguerre M, Klein GJ. Differential sequential septal pacing: a simple maneuver to differentiate nodal versus extranodal ventriculoatrial conduction. Heart Rhythm. 2013;10:1785-1791.

5) Matsushita T, Badhwar N, Collins KK, Van Hare GF, Barbato G, Lee BK, Lee RJ, Scheinman MM. Usefulness of a ventricular extrastimulus from the summit of the ventricular septum in diagnosis of septal accessory pathway in patients with supraventricular tachycardia. Am J Cardiol. 2004;93:643-646.

6) Rosman JZ, John RM, Stevenson WG, Epstein LM, Tedrow UB, Koplan BA, Albert CM, Michaud GF. Resetting criteria during ventricular overdrive pacing successfully differentiate orthodromic reentrant tachycardia from atrioventricular nodal reentrant tachycardia despite interobserver disagreement concerning QRS fusion. Heart Rhythm. 2011;8:2-7. 